\title{
AIAA 99-0156
}

\section{Solving Navier-Stokes Equations} with Advanced Turbulence Models on Three-Dimensional Unstructured Grids

Qunzhen Wang, Steven J. Massey and Khaled S. Abdol-Hamid Analytical Services \& Materials, Inc., Lancaster, CA Neal T. Frink NASA Langley Research Center, Hampton, VA

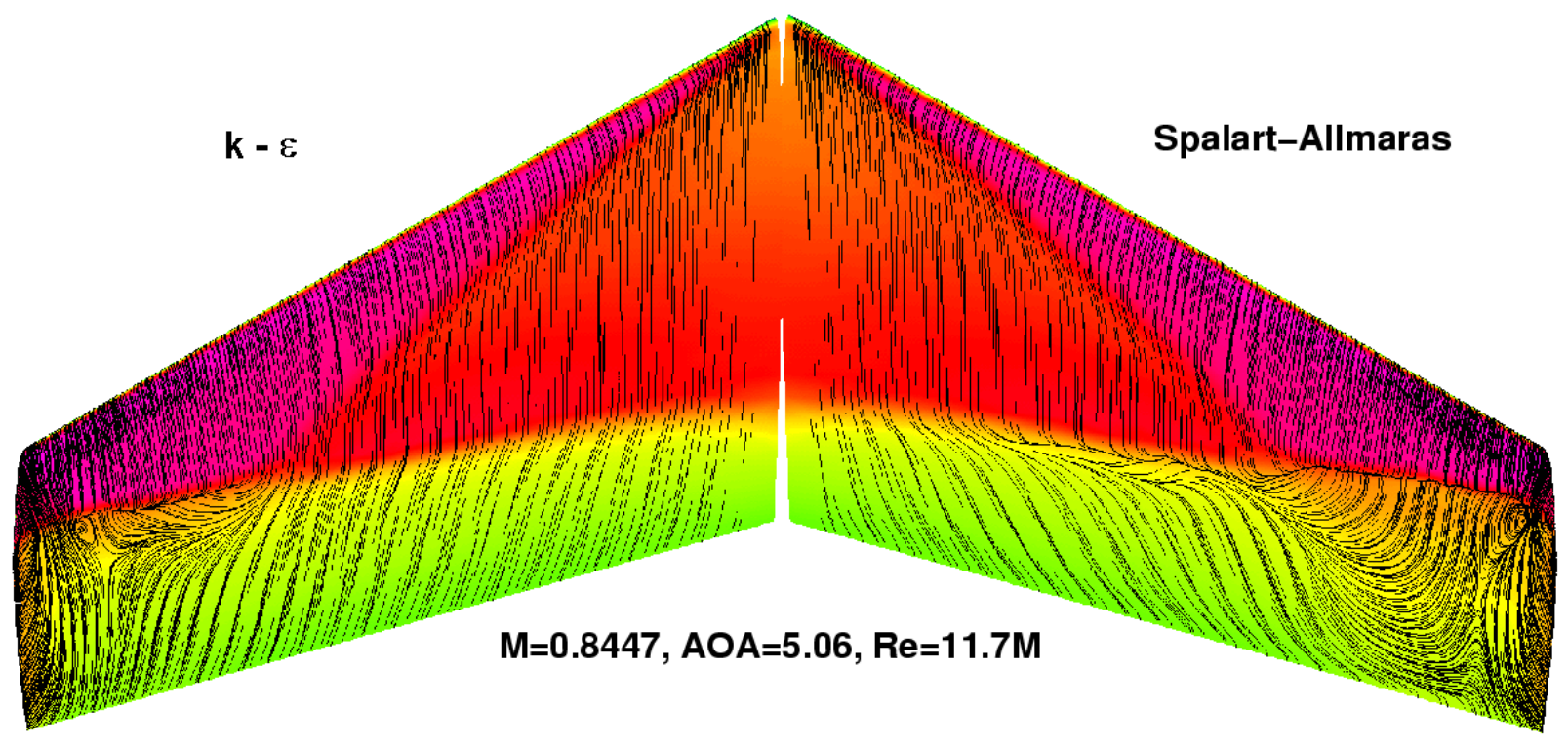

\section{7th AIAA Aerospace Sciences Meeting and Exhibit January 11-14, 1999/Reno, Nevada}




\title{
Solving Navier-Stokes Equations with Advanced Turbulence Models on Three-Dimensional Unstructured Grids
}

\author{
Qunzhen Wang; Steven J. Massey ${ }^{\dagger}$ and Khaled S. Abdol-Hamid ${ }^{\ddagger}$ \\ Analytical Services \& Materials, Inc., Lancaster, CA \\ Neal T. Frink ${ }^{\S}$ \\ NASA Langley Research Center, Hampton, VA
}

\begin{abstract}
USM3D is a widely-used unstructured flow solver for simulating inviscid and viscous flows over complex geometries. The current version (version 5.0) of USM3D, however, does not have advanced turbulence models to accurately simulate complicated flows. We have implemented two modified versions of the original Jones and Launder $k-\varepsilon$ twoequation turbulence model and the Girimaji algebraic Reynolds stress model in USM3D. Tests have been conducted for two flat plate boundary layer cases, a RAE2822 airfoil and an ONERA M6 wing. The results are compared with those of empirical formulae, theoretical results and the existing Spalart-Allmaras one-equation model.
\end{abstract}

\section{Introduction}

$\mathbf{T}$ HE unstructured-grid methodology offers some significant advantages compared to the traditional structured-grid method for simulating flows over complex aerodynamics shapes. This is mainly due to the promise that the construction of an unstructured grid around complex configurations such as an aircraft requires much less time than a comparable block-structured grid. Furthermore, local refinement of unstructured grids can be carried out more easily to improve the accuracy of the simulation. While more work remains to be done to fully realize their potential, much progress has been made in modeling complicated flows on unstructured grid (see Mavriplis ${ }^{1}$ for a review).

One important phenomena for complex viscous flows is turbulence, which is difficult to simulate due to the existence of a wide range of scales. There are many types of method to deal with turbulence, ranging from the simplest algebraic model to the most accurate direct numerical simulation. For most of the turbulence models, the Reynolds stress is assumed to be related to the mean strain rate by the eddy viscosity. Such turbulence models may be divided into zero-equation model (i.e., algebraic model), one-equation model, and two-equation model depending on the number of transport equations need to be solved to obtain the eddy viscosity. The Reynolds stress model does not use the concept of eddy viscosity. Instead, a transport equation for each component of the Reynolds stress tensor

\footnotetext{
* Scientist, Member AIAA. Currently with Thiokol Propulsion, Utah.

†Scientist, Member AIAA.

¥Senior Scientist, Member AIAA.

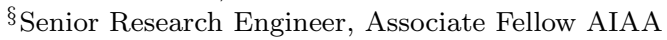

Copyright (C) 1998 by the American Institute of Aeronautics and Astronautics, Inc. All rights reserved.
}

is solved directly. While the above models only solve the mean flow, large eddy simulation (LES) solve the large scale fluctuations in addition to the mean flow and only the effect of small scales (i.e., subgrid scales) are modeled. Finally, the most accurate method is direct numerical simulation (DNS), where both mean flow and all the fluctuations are solved directly.

While the Reynolds stress model, LES and DNS methods are much more accurate than the eddy viscosity based method, they require prohibitive amounts of CPU time and memory. Therefore, the most widely used turbulence models in industry are still those based on the concept of eddy viscosity. This is especially true for unstructured grid CFD codes. For example, the predominantly utilized turbulence model in the finite-element unstructured grid code of Mavriplis $^{2}$ is the algebraic model of Baldwin and Lo$\max ^{3}$ although extension has been made to include a two-equation model (Mavriplis and Martinelli ${ }^{4}$ ). The unstructured nodal-based finite volume code FUN3D (see Anderson, ${ }^{5}$ Anderson and Bonhaus ${ }^{6}$ ), contains two one-equation models, one by Baldwin and Barth ${ }^{7}$ and the other by Spalart and Allmaras. ${ }^{8}$

USM3D is a tetrahedral cell-centered unstructured flow solver for simulating inviscid and viscous flows over complex geometries. It is developed by Frink ${ }^{9-12}$ at NASA Langley Research Center and is now being widely used in both industry and government. This code is part of the TetrUSS flow analysis and design package which won the 1996 NASA Software of the Year award. The algorithm of USM3D consists of a cell-centered, upwind-biased, finite-volume, implicit/explicit algorithm capable of solving the compressible Euler and Navier-Stokes equations on unstructured tetrahedral meshes. Like most other unstructured CFD codes, however, USM3D does not 
have advanced turbulence models to accurately simulate complex flows. The current production version of the code only has the one-equation turbulence model developed by Spalart and Allmaras, ${ }^{8}$ although a two equation $k-\varepsilon$ model was implemented in a previous research version of USM3D (Kwon and $\mathrm{Hah}^{13}$ ). It is well known that one-equation turbulence models are not adequate for complex flows such as separated flows or shear flows. The objective of this study is to compare the performance of the recently added advanced turbulence models (see Wang et al. ${ }^{14}$ ) with experimental data, theoretical results and the results from the existing Spalart-Allmaras one-equation model implemented by Frink. ${ }^{12}$

\section{Governing Equations}

The integral form of the Navier-Stokes equations, which govern the compressible, Newtonian flow of fluid in the absence of external forces, can be written as

$$
\frac{\partial}{\partial t} \iiint \mathbf{Q} d V+\iint \mathbf{F}(\mathbf{Q}) \cdot \hat{n} d S=\iint \mathbf{G}(\mathbf{Q}) \cdot \hat{n} d S
$$

where the first term (time changing rate) is integrated over the volume of a bounded domain while the second term (convection) and the third term (diffusion) are integrated over the boundary of this domain. The quantities $\mathbf{Q}, \mathbf{F}(\mathbf{Q})$, and $\mathbf{G}(\mathbf{Q})$ are vectors with five components and Eq. (1) contains five equations corresponding to the conservation of mass, momentum, and energy. The unknowns in Eq. (1) are

$$
\mathbf{Q}=\left[\begin{array}{c}
\rho \\
\rho u \\
\rho v \\
\rho w \\
e
\end{array}\right]
$$

where $\rho, u, v, w$ and $e$ are density, three velocity components, and total energy, respectively. The inviscid flux is

$$
\mathbf{F}(\mathbf{Q}) \cdot \hat{n}=\left[\begin{array}{c}
\rho \\
\rho u \\
\rho v \\
\rho w \\
e+p
\end{array}\right]+p\left[\begin{array}{c}
0 \\
\hat{n}_{x} \\
\hat{n}_{y} \\
\hat{n}_{z} \\
0
\end{array}\right]
$$

where $p$ is the pressure and $\hat{n}_{x}, \hat{n}_{y}$, and $\hat{n}_{z}$ are Cartesian components of the exterior surface unit normal $\hat{n}$ on the boundary of the domain. The viscous flux is

$$
\mathbf{G}(\mathbf{Q}) \cdot \hat{n}=\frac{M_{\infty}}{R e_{L}}\left(\hat{n}_{x} G_{1}+\hat{n}_{y} G_{2}+\hat{n}_{z} G_{3}\right)
$$

where

$$
G_{i}=\left[\begin{array}{c}
0 \\
\tau_{i 1} \\
\tau_{i 2} \\
\tau_{i 3} \\
u_{j} \tau_{i j}-q_{i}
\end{array}\right]
$$

$M_{\infty}$ is the free-stream Mach number and $R e_{L}$ is the Reynolds number based on a typical length scale (e.g., the total length of the flat plate).

The total stress $\tau_{i j}$ and heat flux $q_{i}$ can be divided into a laminar part (denoted by superscript $L$ ) and a turbulence part (denoted by superscript $T$ )

$$
\begin{gathered}
\tau_{i j}=\tau_{i j}^{L}+\tau_{i j}^{T} \\
q_{i}=q_{i}^{L}+q_{i}^{T}
\end{gathered}
$$

with the laminar part being

$$
\begin{gathered}
\tau_{i j}^{L}=\mu^{L}\left[\left(\frac{\partial u_{i}}{\partial x_{j}}+\frac{\partial u_{j}}{\partial x_{i}}\right)-\frac{2}{3} \delta_{i j} \frac{\partial u_{k}}{\partial x_{k}}\right] \\
q_{i}^{L}=-\frac{\mu^{L}}{(\gamma-1) \operatorname{Pr}^{L}} \frac{\partial T}{\partial x_{i}}
\end{gathered}
$$

where $\mu^{L}$ is the molecular viscosity, $T$ is the temperature, $\operatorname{Pr}^{L}$ is the molecular Prandtl number, and $\gamma=1.4$ is the ratio of specific heats. Following Eqs. (8) and (9), the Reynolds stress and turbulent heat flux can be approximated as

$$
\begin{gathered}
\tau_{i j}^{T}=\mu^{T}\left[\left(\frac{\partial u_{i}}{\partial x_{j}}+\frac{\partial u_{j}}{\partial x_{i}}\right)-\frac{2}{3} \delta_{i j} \frac{\partial u_{k}}{\partial x_{k}}\right]-\frac{2}{3} \rho k \delta_{i j} \\
q_{i}^{T}=-\frac{\mu^{T}}{(\gamma-1) \operatorname{Pr}^{T}} \frac{\partial T}{\partial x_{i}}
\end{gathered}
$$

Note that the last term in Eq. (10) is presented only when a transport equation for turbulent kinetic energy $k$ is solved.

\section{Transport Equations for $k$ and $\varepsilon$}

In the $k-\varepsilon$ model, two transport equations, one for turbulent kinetic energy $k$ and the other for dissipation rate $\varepsilon$, are solved and the eddy viscosity $\mu^{T}$ in Eqs. (10) and (11) are then calculated based on $k$ and $\varepsilon$

$$
\mu^{T}=C_{\mu} f_{\mu} \rho \frac{k^{2}}{\varepsilon}
$$

where $f_{\mu}$ is a damping function and $C_{\mu}=0.09$. The transport equation for $k$ is

$$
\frac{\partial \rho k}{\partial t}+\frac{\partial \rho k u_{j}}{\partial x_{j}}-\frac{\partial}{\partial x_{j}}\left[\mu_{k} \frac{\partial k}{\partial x_{j}}\right] \frac{M_{\infty}}{R e_{L}}=S_{k}
$$

where

$$
\begin{gathered}
S_{k}=\bar{P} \frac{M_{\infty}}{R e_{L}}-\rho(1+\Gamma) \varepsilon \frac{R e_{L}}{M_{\infty}} \\
\bar{P}=\tau_{i j}^{T} \frac{\partial u_{i}}{\partial x_{j}} \\
\mu_{k}=\mu^{L}+\frac{\mu^{T}}{\sigma_{k}} \quad \sigma_{k}=1.0
\end{gathered}
$$

Similarly, the transport equation for $\varepsilon$ can be written as

$$
\frac{\partial \rho \varepsilon}{\partial t}+\frac{\partial \rho \varepsilon u_{j}}{\partial x_{j}}-\frac{\partial}{\partial x_{j}}\left[\mu_{\varepsilon} \frac{\partial \varepsilon}{\partial x_{j}}\right] \frac{M_{\infty}}{R e_{L}}=S_{\varepsilon}
$$




$$
\begin{gathered}
S_{\varepsilon}=C_{\varepsilon 1} \bar{P} \frac{\varepsilon}{k} \frac{M_{\infty}}{R e_{L}}-C_{\varepsilon 2} f_{2} \frac{\varepsilon}{k} \frac{R e_{L}}{M_{\infty}}\left[\rho \varepsilon-L_{k}\left(\frac{M_{\infty}}{R e_{L}}\right)^{2}\right] \\
L_{k}=2 \mu^{L}\left[\left(\frac{\partial \sqrt{k}}{\partial x}\right)^{2}+\left(\frac{\partial \sqrt{k}}{\partial y}\right)^{2}+\left(\frac{\partial \sqrt{k}}{\partial z}\right)^{2}\right] \\
\mu_{\varepsilon}=\mu^{L}+\frac{\mu^{T}}{\sigma_{\varepsilon}} \quad \sigma_{\varepsilon}=1.3 \quad C_{\varepsilon 1}=1.44 \quad C_{\varepsilon 2}=1.92 \\
f_{2}=1-0.3 \exp \left(-R_{t}^{2}\right) \quad R_{t}=\frac{\rho k^{2}}{\mu^{L} \varepsilon}
\end{gathered}
$$

In all of the equations, as well as, all the figures shown in this paper, unless explicitly stated otherwise, the length is normalized by a characteristic length $L$, the velocity by $a_{\infty}$, the density by $\rho_{\infty}$, the viscosity by $\mu_{\infty}$, the turbulent kinetic energy $k$ by $a_{\infty}^{2}$, and the dissipation rate $\varepsilon$ by $\rho_{\infty} a_{\infty}^{4} / \mu_{\infty}$, where $a_{\infty}$ is the freestream speed of sound, $\rho_{\infty}$ is the free-stream density, and $\mu_{\infty}$ is the free-stream molecular viscosity.

The compressibility correction $\Gamma$ in Eq. (14) and the damping function $f_{\mu}$ in Eq. (12) can take different forms. The two most widely used compressibility corrections are Sarkar et al. ${ }^{15}$ model

$$
\Gamma=M_{t}^{2}
$$

and Wilcox ${ }^{16}$ model

$$
\Gamma=\left(M_{t}^{2}-M_{t 0}^{2}\right) H\left(M_{t}-M_{t 0}\right)
$$

where $H(x)$ is the Heaviside step function, the turbulent Mach number $M_{t}=\sqrt{k} / a$ with $a$ being the local speed of sound. The damping function could take one of the following three forms: a) Jones and Launder ${ }^{17}$ form

$$
f_{\mu}=\exp \left[-\frac{3.41}{\left(1+\frac{R_{t}}{50}\right)^{2}}\right]
$$

b) Van Driest form (Nagano and Hishida ${ }^{18}$ )

$$
f_{\mu}=1-\exp \left(\frac{-n^{+}}{A^{+}}\right)
$$

c) Speziale et $a l .{ }^{19}$ ) form

$$
f_{\mu}=\left(1+\frac{3.45}{\sqrt{R_{t}}}\right) \tanh \left(\frac{n^{+}}{70}\right)
$$

For the results shown in this paper, ivisc $=6$ refers to the Jones and Launder form of the damping function, Eq. (24), with no compressibility correction (i.e., $\Gamma=$ $0)$ and ivisc $=7$ refers to the Jones and Launder model modified by Carlson ${ }^{20}$ as given in Eqs. (27-31).

$$
\begin{gathered}
\kappa=0.41 \quad \alpha=1.15 \quad C_{\varepsilon 2}=1.9 \\
C_{\varepsilon 1}=\alpha\left(1+0.2174 \frac{P}{\varepsilon}\right)
\end{gathered}
$$

$$
\begin{aligned}
C_{1} & =\alpha\left(1+0.2174 \frac{1.5}{\varepsilon}\right) \\
\sigma_{\varepsilon} & =\frac{\kappa^{2}}{\left(C_{\varepsilon 2}-C_{1}\right) \sqrt{C_{\mu}}} \\
f_{\mu} & =\exp \left[-\frac{6}{\left(1+\frac{R_{t}}{75}\right)^{2}}\right]
\end{aligned}
$$

\section{Algebraic Reynolds Stress Model}

The $k-\varepsilon$ model discussed above is the standard model, which is also called the "linear model" because the turbulent stress is linearly related to the mean strain rate by the eddy viscosity as is clear from Eq.(10). However, various direct numerical simulation (DNS) data have shown that the turbulent stress does not vary linearly with the mean strain rate. A more accurate model is the Reynolds stress model where a transport equation for each component of the Reynolds stress tensor is solved directly and the concept of eddy viscosity is not used. However, the Reynolds stress model requires a tremendous amount of CPU time and memory and, thus, is seldom used in real complex engineering applications. Something in between is the algebraic stress model, in which a nonlinear term is added to the turbulent stress (so it is also called the "nonlinear model"). Following Girimaji, ${ }^{21}$ the turbulent stress in the algebraic stress model is

$$
\begin{aligned}
\tau_{i j}^{T}= & \mu^{T}\left[S_{i j}-\frac{1}{3} \delta_{i j} \frac{\partial u_{k}}{\partial x_{k}}\right]-\frac{2}{3} \rho k \delta_{i j} \\
& +2 \mu^{T} K_{1} \frac{k}{\varepsilon}\left[S_{i k} W_{k j}-W_{i k} S_{k j}\right] \\
& +2 \mu^{T} K_{1} \frac{k}{\varepsilon}\left[S_{i k} S_{k j}-\frac{1}{3} S_{k l} S_{l k}\right]
\end{aligned}
$$

where the mean strain rate and mean vorticity are

$$
\begin{aligned}
S_{i j} & =\frac{1}{2}\left(\frac{\partial u_{i}}{\partial x_{j}}+\frac{\partial u_{j}}{\partial x_{i}}\right) \\
W_{i j} & =\frac{1}{2}\left(\frac{\partial u_{i}}{\partial x_{j}}-\frac{\partial u_{j}}{\partial x_{i}}\right)
\end{aligned}
$$

respectively. In Eq.(32), $K_{1}$ and $K_{2}$ are given by

$$
K_{1}=\frac{G_{2}}{G_{1}}
$$

and

$$
K_{2}=\frac{G_{3}}{G_{1}}
$$

while $G_{1}$ can be obtained from

$$
G_{1}=\left\{\begin{array}{lr}
\frac{-L_{1}^{0} L_{2}}{\left(L_{0}^{1}\right)^{2}+2 \eta_{2}\left(L_{4}\right)^{2}}, & \eta_{1}=0 \\
\frac{-L_{1}^{0} L_{2}}{\frac{2}{3} \eta_{1}\left(L_{3}\right)^{2}+\left(L_{0}^{1}\right)^{2}+2 \eta_{2}\left(L_{4}\right)^{2}}, & L_{1}^{1}=0 \\
\frac{p}{3}+\left(-\frac{b}{2}+\sqrt{D}\right)^{\frac{1}{3}}+\left(-\frac{b}{2}-\sqrt{D}\right)^{\frac{1}{3}}, & D>0 \\
\frac{p}{3}+2 \sqrt{-\frac{a}{3}} \cos \left(\frac{\theta}{3}\right), & D<0, b<0 \\
\frac{p}{3}+2 \sqrt{-\frac{a}{3}} \cos \left(\frac{\theta}{3}+\frac{2 \pi}{3}\right), & D<0, b>0
\end{array}\right.
$$


and $G_{2}$ and $G_{3}$ are

$$
\begin{aligned}
& G_{2}=\frac{-L_{4} G_{1}}{L_{0}^{1}-\eta_{1} L_{1}^{1} G_{1}} \\
& G_{3}=\frac{2 L_{3} G_{1}}{L_{0}^{1}-\eta_{1} L_{1}^{1} G_{1}}
\end{aligned}
$$

where

$$
\begin{aligned}
& \eta_{1}=\frac{k^{2}}{\varepsilon} S_{i j} S_{i j}\left(\frac{M_{\infty}}{R e_{L}}\right)^{2} \\
& \eta_{2}=\frac{k^{2}}{\varepsilon} W_{i j} W_{i j}\left(\frac{M_{\infty}}{R e_{L}}\right)^{2} \\
& p=\frac{2 L_{1}^{0}}{\eta_{1} L_{1}^{1}} \\
& r=\frac{L_{2} L_{1}^{0}}{\left(\eta_{1} L_{1}^{1}\right)^{2}} \\
& q=\frac{\left(L_{1}^{0}\right)^{2}+\eta_{1} L_{2} L_{1}^{1}-\frac{2}{3} \eta_{1}\left(L_{3}\right)^{2}+2 \eta_{2}\left(L_{4}\right)^{2}}{\left(\eta_{1} L_{1}^{1}\right)^{2}} \\
& a=q-\frac{p^{2}}{3} \\
& b=\frac{1}{27}\left(2 p^{3}-9 p q+27 r\right) \\
& D=\frac{b^{2}}{4}+\frac{a^{3}}{27} \\
& \cos \theta=\frac{-b / 2}{\sqrt{-a^{3} / 27}} \\
& L_{0}^{1}=\frac{C_{1}^{0}}{2}-1 \\
& L_{1}^{1}=C_{1}^{1}+2 \\
& L_{2}=\frac{C_{2}}{2}-\frac{2}{3} \\
& L_{3}=\frac{C_{3}}{2}-1 \\
& L_{4}=\frac{C_{4}}{2}-1 \\
& C_{1}^{0}=3.4 \quad C_{1}^{1}=1.8 \\
& C_{2}=0.36 \quad C_{3}=1.25 \quad C_{4}=0.4
\end{aligned}
$$

Furthermore, instead of $C_{\mu}=0.09$ for the linear model, $C_{\mu}=-G_{1}$ is applied in the nonlinear model.

\section{Numerical Procedure}

The details of the numerical procedure for solving equation (1) are discussed in Frink ${ }^{10-12}$ and only a brief overview is given here. The spatial computational domain is divided into a finite number of tetrahedral cells and a finite-volume discretization is applied to each cell. This procedure results in a set of volume-averaged state variables $\mathbf{Q}$ which are in balance with the area-averaged inviscid flux $\mathbf{F}(\mathbf{Q})$ and viscous flux $\mathbf{G}(\mathbf{Q})$. Inviscid fluxes are obtained across each cell face using either the Roe ${ }^{22}$ flux-difference splitting approach or the Van Leer $^{23}$ flux-vector splitting technique. The data at nodal points could be obtained from the cell-averaged data by either an inversedistance weighted averaging scheme or a Laplacianweighted averaging scheme. The viscous fluxes are approximated at the cell-face centroids by linear reconstruction. An implicit time integration algorithm using the linearized backward Euler time differencing approach is applied to update the solution. The resulting linear system of equations are solved at each time step with a subiterative procedure by a point-Jacobi method. Convergence to the steady state solution is accelerated by advancing the equations at each cell in time by the maximum permissible time step in that cell. The CFL number is scaled according to the deviation of cell aspect ratio from the ideal value of an isotropic tetrahedron.

The solution procedure for the two turbulence transport equations (13) and (17) are similar to that for solving the Navier-Stokes equations. Equations (13) and (17) are solved separately from the flow governing equations and from each other using the same backward Euler time integration scheme. The $k$ and $\varepsilon$ equations can be solved using either first-order or second-order schemes. For the second-order method, either Roe's SuperBee limiter or the Minimum Modulus (Min-Mod) limiter could be applied. To allow a large CFL number, implicit method is used to solve the $k$ and $\varepsilon$ equations.

Two input parameters $k_{0}$ and $\mu_{0}^{T}$ are needed to specify the initial conditions and limit the smallest values of $k$ and $\varepsilon$. The initial conditions are $k=k_{0}$ and $\varepsilon=\varepsilon_{0}=C_{\mu} \rho k_{0}^{2} / \mu_{0}^{T}$. The turbulent kinetic energy and dissipation rate are not allowed to be smaller than $k_{0}$ and $\varepsilon_{0}$, respectively. On solid surfaces, the boundary conditions are $k=k_{0}$ and $\varepsilon=L_{k} / \rho\left(M_{\infty} / R e_{L}\right)^{2}$. Far field boundary conditions are applied by extrapolating $k$ and $\varepsilon$ from the interior for outflow boundaries and taken from the free-stream for the inflow boundaries.

The $k-\varepsilon$ model has been coupled with wall function formulations to reduce the need for resolving the flow in the near wall region. The following three different wall functions could be used together with the $k-\varepsilon$ model: (1) iwallf=1: the original wall function developed by Frink; ${ }^{12}(2)$ iwallf=2: a wall function similar to that used in PAB3D (see Abdol-Hamid et al. ${ }^{24}$ for 
details); (3) iwallf=3: same as the original wall function except the velocity components are zero at the solid surface. For iwallf=1, a slip velocity boundary condition is obtained by solving the Spalding formula

$y^{+}=u^{+}+e^{-\kappa B}\left[e^{\kappa u^{+}}-1-\kappa u^{+}-\frac{\left(\kappa u^{+}\right)^{2}}{2}-\frac{\left(\kappa u^{+}\right)^{3}}{6}\right]$

with $\kappa=0.4$ and $B=5.5$ using Newton-Raphson iteration method while the velocity at the solid boundary for $i w a l l f=2$ and $i w a l l f=3$ are zero.

\section{Results}

\section{Flat Plate Cases: BLT2 and BLT3}

Two tetrahedral grids for a simple flat plate boundary layer have been generated using the grid generator VGRID (Pirzadeh ${ }^{25}$ ). For both grids, results of the newly added $k-\varepsilon$ models are compared with the existing Spalart-Allmaras model and with experimental and theoretical data. The computational domain is from $x=-0.5$ to $x=1.0$ in the streamwise direction, from $y=-0.02$ to $y=0.02$ in the spanwise direction $(-0.05<y<0.05$ for BLT2), and from $z=0$ to $z=0.22$ in the wall normal direction. The free-stream Mach number and Reynolds number are $M_{\infty}=0.5$, $R e_{L}=2 \times 10^{6}$, respectively. The grid characteristics for the flat plate cases are summarized in Table 1.

Table 1 Characteristics of flat plate grids.

\begin{tabular}{cccccc}
\hline \hline Case & Cells & Nodes & Faces & B.Nds & B.Fcs \\
\hline BLT2 & 48,497 & 9,629 & 99,805 & 2,813 & 5,612 \\
BLT3 & 37,483 & 8,038 & 78,328 & 3,364 & 6,724 \\
\hline \hline
\end{tabular}

The grids for the flat plate cases are shown in Figure 1. There are four cells in the spanwise direction for BLT2 and two cells for the BLT3 grid. Near the wall, the grid spacings for the BLT3 grid are much smaller than that for BLT2 but the grid spacings for BLT3 is much larger than that for BLT2 far away from the wall. For the BLT3 grid, the first node point away from the wall has a $y^{+} \approx 1.8$, while for the BLT2 grid $y^{+} \approx 223$. Therefore, a wall function has to be applied for BLT2 while no wall function is needed for the BLT3 grid.

\section{BLT2 Flat Plate - Wall Function Case}

Results using the Spalart-Allmaras and linear $k-\varepsilon$ models are shown in Figure 2. The residual history for the Spalart-Allmaras and Carlson modified $k-\varepsilon$ model both converge rapidly at nearly the same rate to a level of 9 orders of magnitude smaller than the initial residual. The first linear model (ivisc=6) converged much slower after the $500^{t h}$ time step and only decreased by less than 4 orders of magnitude after 4,000 time steps. The CFL number is allowed to increases dynamically from 1 to 200 according to the residual: it increases when the residual is decreasing and decreases when the residual is increasing. For each of the three

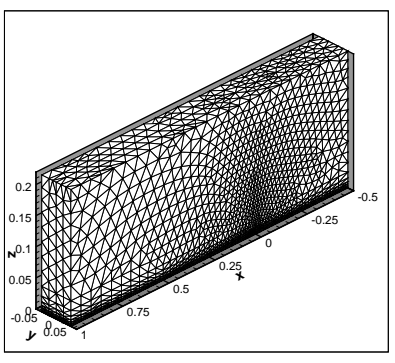

a) Full BLT2 grid.

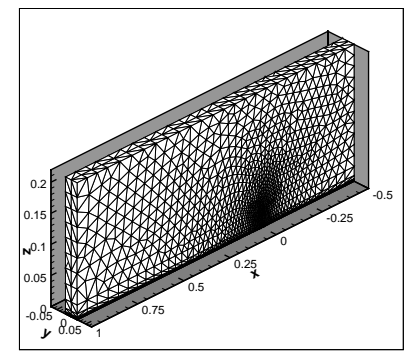

c) Full BLT3 grid.

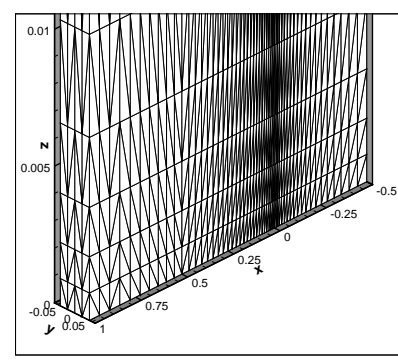

b) Wall of BLT2 grid.

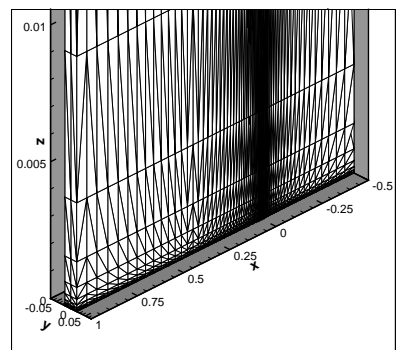

d) Wall of BLT3 grid.
Fig. 1 Whole domain and near wall regions of BLT2 and BLT3 grids for a flat plate boundary layer.

models tested, the CFL number rapidly increased to its maximum value within 40 time steps and remained at 200 for the duration of the run, indicating a robust convergence. The velocity profile is compared with the empirical formula of Spalding, Eq. (56), while the skin friction coefficient is compared with the theoretical values for fully turbulent flow.

$$
C_{f}=0.0583\left(\operatorname{Re}_{x}\right)^{-1 / 5}
$$

It is evident that $u^{+}$from the second (ivisc=7) $k$ $\varepsilon$ model is the closest to Spalding's formula with the Spalart-Allmaras results the next closest. The first $k-\varepsilon$ model initially matches the data as dictated by the wall function, but then significantly under predicts $u^{+}$. The skin friction coefficient from the first $k-\varepsilon$ model is significantly larger than the theoretical value whereas the Spalart-Allmaras model gives a result slightly smaller than the theoretical value and the second $k$ - $\varepsilon$ model initially matches theory closely before predicting a slightly larger value. In summary, for the coarse flat plate grid using a wall function, the Carlson modified linear $k-\varepsilon$ model performed best, with the Spalart-Allmaras model nearly as good and the first $k-\varepsilon$ model not in good agreement with empirical or theoretical data. The CPU time per timestep per cell on an Intel Pentium II $300 \mathrm{MHz}$ was $229 \mu \mathrm{sec}$ for the Spalart-Allmaras model and $13 \%$ more for the $k-\varepsilon$ models.

\section{BLT3 Flat Plate - Grid Resolved Case}

The results from the Spalart-Allmaras and $k-\varepsilon$ models are shown in Figure 3. The residual history for all three models converge rapidly at roughly the same rate to a level of 5 orders of magnitude smaller than the 


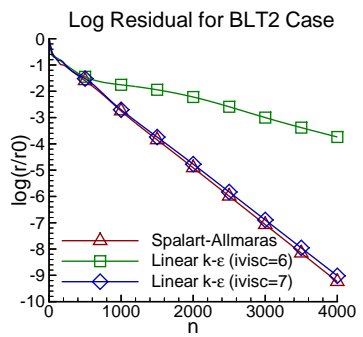

a)

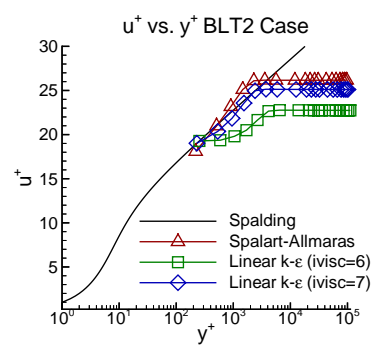

c)

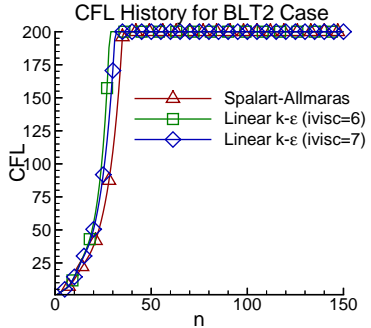

b)

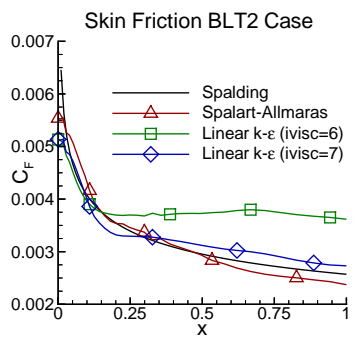

d)

Fig. 2 History of a) Log residual and b) CFL number. Converged c) streamwise velocity component in wall coordinates at $x / c=0.5$ and d) skin friction coefficient. BLT2 case.

initial residual. The temporary hump in the residual trace around $n=1,000$ is characteristic of the establishment of turbulence for a non-wall function grid. For each of the three models tested, the CFL number rapidly increased to its maximum value within 75 time steps and remained at 200 for the duration of the run, indicating a robust convergence.

Inspection of the velocity profile and skin friction plots, see Figure 3, confirms that for the BLT3 grid a wall function is not needed. It is evident that $u^{+}$from the Spalart-Allmaras model is closer to the Spalding curve with the second $k-\varepsilon$ model under predicting $u^{+}$ slightly and the first $k-\varepsilon$ model under prediction significantly. The skin friction coefficient from the first $k$ - $\varepsilon$ model is significantly larger than the theoretical value whereas the Spalart-Allmaras model gives a result slightly smaller than the theoretical value and the second $k-\varepsilon$ model matches theory the closest predicting a slightly larger value.

In summary, for the BLT3 refined flat plate grid without using a wall function, the Carlson modified $k-\varepsilon$ model performed best for predicting skin friction and is a close second to Spalart-Allmaras for velocity profile. Experience with the linear models in the structured code PAB3D ${ }^{24}$ indicates that with grid refinement the accuracy of the linear models will improve significantly. The CPU time per timestep per cell on an SGI with two IP30 processors was $194 \mu \mathrm{sec}$ for the Spalart-Allmaras model and $10 \%$ more for the $k-\varepsilon$ models.

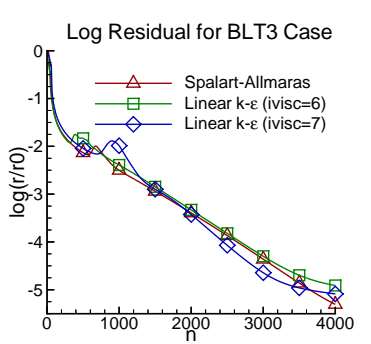

a)

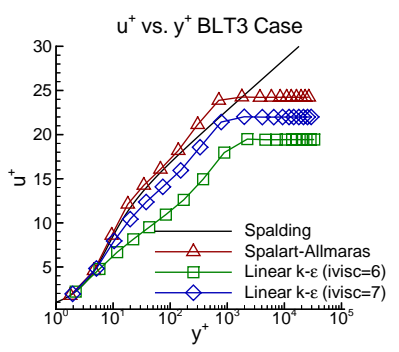

c)

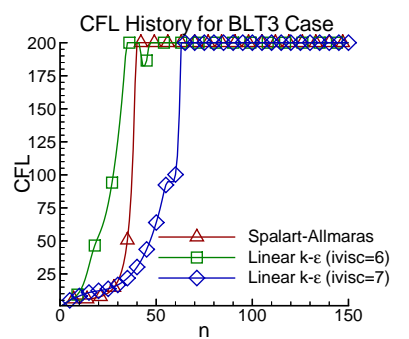

b)

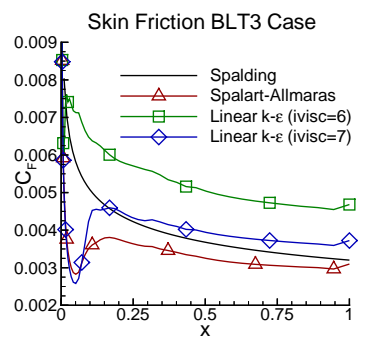

d)
Fig. 3 History of a) Log residual and b) CFL number. Converged c) streamwise velocity component in wall coordinates at $x / c=0.5$ and d) skin friction coefficient. BLT3 case.

\section{RAE2822 Airfoil}

In this section results for the previously mentioned turbulence models of Spalart-Allmaras model, $k-\varepsilon$ (ivisc=6), $k-\varepsilon$ (ivisc=7) will be compared with the Girimaji algebraic Reynolds stress model (ARSM) as well as the experimental results of case 10 of Cook et $a l .{ }^{26}$ for a transonic airfoil.

The computational domain extends 6 chord lengths away from the airfoil in all directions with a grid width $0.2 c$ spanning 2 cells. The free-stream Mach number of the boundary layer, Reynolds number and corrected free air angle-of-attack is $M_{\infty}=0.75, R e=6.2 \times 10^{6}$ and $\alpha=2.81^{\circ}$ respectively. The grid consist of 29,976 cells, 8,477 nodes, 66,772 faces, 6,820 boundary nodes and 13,640 boundary faces. At the first node away from the wall $y^{+} \approx 0.8$. Full and closeup views of the grid are shown in Figure 4.

Unlike the previous flat plate cases, the residual and CFL history (Figure 5) are much more noisy, which may be due to unsteadiness in the region behind the shock. All of the models converge between 3 and 3.5 orders of magnitude after 4,000 time steps. Initially the ARSM shows a great deal of oscillation since it is more dependent on the initial condition. Also observed is a large increase in residual for the second linear model before finally settling down. The CFL history reveals that for the first 400 time steps each model is behaving similarly with the exception of the ARSM whose CFL history appears to be shifted to the left. After $n=400$ the CFL number for all models oscillate periodically at high frequency.

From the lift and drag coefficient history plots (Fig- 
ure 5) it is evident that the Spalart-Allmaras, first $k-\varepsilon$ and Girimaji models follow the same convergence trend while the second linear model oscillates much more before settling down at around $n=2,500$. The results at $n=4,000$ along with experimental data and results from PAB3D for the first linear model are compared in Table 2. Final results show that the Spalart-Allmaras model is in closest agreement with experimental data. The next best results for USM3D are produced by the first linear model, the ARS model and finally the second linear model. As an indicator of grid dependence for the advanced models, results in good agreement with experimental data from PAB3D are shown for the first linear model.

Table 2 Comparison of $C_{L}$ and $C_{D}$ with experimental data of Cook et al. ${ }^{26}$ and PAB3D linear $k-\varepsilon$ model (ivisc $=6) .{ }^{24}$

\begin{tabular}{ccccc}
\hline \hline Case & $C_{L}$ & $\%$ diff & $C_{D}$ & \% diff \\
\hline Experiment & 0.743 & - & 0.0242 & - \\
Spalart-Allmaras & 0.732 & -1.5 & 0.0214 & -11.6 \\
$k-\varepsilon$ (ivisc=6) & 0.691 & -7.0 & 0.0199 & -17.8 \\
$k-\varepsilon$ (ivisc=7) & 0.649 & -12.7 & 0.0178 & -26.4 \\
Girimaji ARSM & 0.803 & 8.1 & 0.0293 & 21.1 \\
PAB3D $k-\varepsilon$ & 0.720 & -3.1 & 0.0257 & 6.2 \\
\hline \hline
\end{tabular}

In Figure 6 the predicted coefficient of pressure from each model is plotted with the experimental data of Cook. ${ }^{26}$ All models miss the location of the first suction peak by approximately $2 \% \mathrm{c}$. This is likely due to the fact that the current implementation of the turbulence models in USM3D does not allow for setting a trip location for the boundary layer, while in the experiment it was set to $3 \%$ c. Other areas of disagreement with experiment and among the models themselves is the shock location and region aft of the shock. The prediction of shock location by the second linear model was in excellent agreement while the other models all predicted different locations-all aft of the experimental location. Following the shock, all models predicted a lower $C_{P}$ than experiment, with the Girimaji model in the best agreement.

The CPU time per timestep per cell on an Intel Pentium II $300 \mathrm{MHz}$ was $207 \mu$ sec for the Spalart-Allmaras model, $224 \mu \mathrm{sec}$ for the first $k-\varepsilon$ model, $234 \mu \mathrm{sec}$ for the second $k-\varepsilon$ model and $302 \mu$ sec for the Girimaji model.

\section{ONERA M6 Wing}

The tetrahedral viscous grid for the ONERA M6 wing was generated using VGRID and is similar in construction to those in Frink. ${ }^{12}$ The grid consist of 338,417 cells, 59,496 nodes, 682,257 faces, 5,425 boundary nodes and 10,846 boundary faces. On the wing surface $y^{+} \approx 2$ for $M_{\infty}=0.8447, R e_{\text {mac }}=$ $11.7 \times 10^{6}$ and $\alpha=5.06^{\circ}$. The computational domain is bounded by a rectangular box defined by

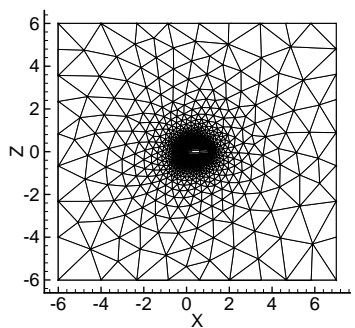

a)

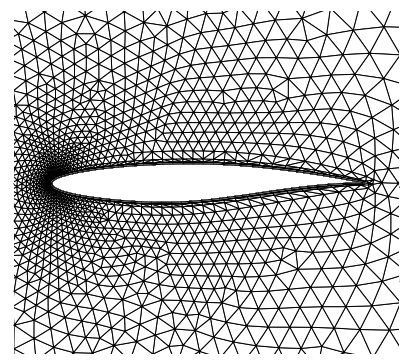

b)
Fig. 4 RAE2822 airfoil grid. a) whole domain; b) near airfoil.

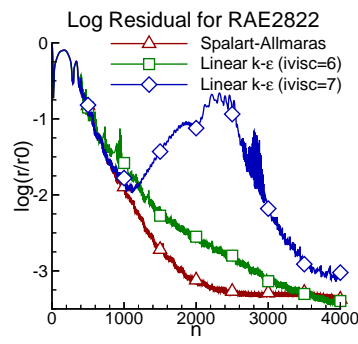

a)

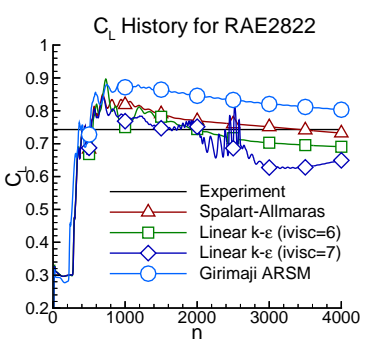

c)

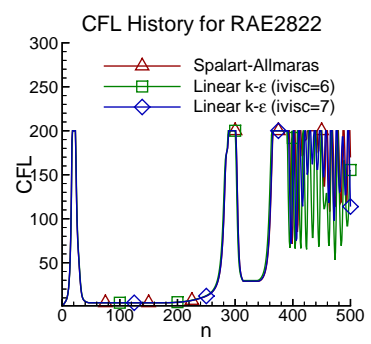

b)

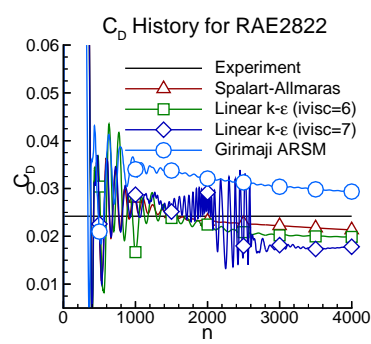

d)
Fig. 5 History of a) Log residual, b) CFL number, c) $C_{L}$ and d) $C_{D}$ for RAE2822 airfoil.

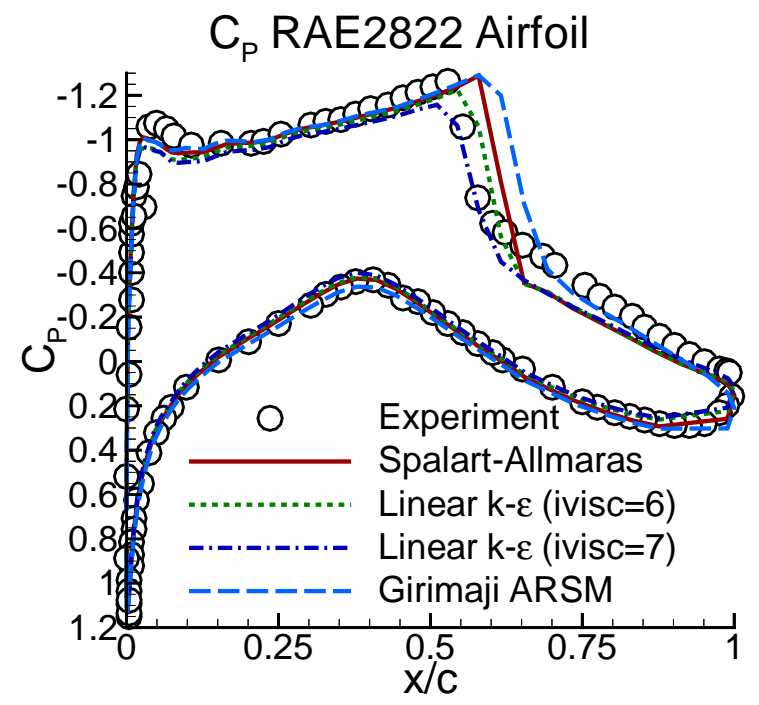

Fig. 6 Comparison of coefficient of pressure from each model $(n=4,000)$ with the experimental data of $\mathrm{Cook}^{26}$. 
$-6.5 \leq x \leq 6.5,0 \leq y \leq 4$, and $-6.5 \leq z \leq 6.5$, in aerodynamic coordinates relative to a semispan length of 1 . Surface and symmetry plane meshes are shown in Figure 7.

For this ONERA M6 wing case, results are presented for the Spalart-Allmaras model and the second linear (ivisc=7) model with varying numbers of Jacobi iterations in the solution of the $k-\varepsilon$ equations. In Figure 8 , the residual history is seen to be fairly similar among the models with the Spalart-Allmaras being the smoothest and both linear cases being more noisy until $n=500$. To aid solution stability, the original CFL ramping scheme in USM3D was used to bring the CFL number to a constant value of 75 , rather than letting it vary dynamically up to 200 as in previous cases.

The coefficient of lift and drag histories (Figure 8) confirm that the reduction in the number of iterations on the $k-\varepsilon$ equations causes no inaccuracy in the solution. This is significant because the reduction brings a $10 \%$ time savings. While the two linear cases agree with each other, the Spalart-Allmaras model predicts a $4 \%$ lower $C_{L}$ while agreeing exactly with the linear models prediction of $C_{D}$. The downward trend in both $C_{L}$ and $C_{D}$ plots indicate a slight lack of convergence despite the drop in log residual of over 3 orders of magnitude. This may be a result of the unsteadiness of the flow field.

Limiting surface streamlines simulating "oil-flow" patterns shown in Figure 9 show a significant amount of shock separated flow beyond the $\eta \approx 0.65$ for both models, with the Spalart-Allmaras model results showing the strongest separation. The $C_{P}$ plots in Figure 10 demonstrate this with the exception of the $\eta=0.90$ plane where Spalart-Allmaras matches the shock location well. It should be noted however, that the solutions in the tip region, $\eta>0.90$, can be particularly sensitive to a variety of factors such as grid density and turbulence model (see Rumsey and Vatsa ${ }^{27}$ ), and should be explored further in future studies.

The CPU time per timestep per cell on an SGI with two IP30 processors was $216 \mu$ sec for the SpalartAllmaras model, $223 \mu \mathrm{sec}$ for the second (ivisc $=7$ ) $k-\varepsilon$ model with 2 Jacobi iterations (nstagek $=2$ ) on the $k$ $\varepsilon$ equations and $249 \mu \mathrm{sec}$ for the second linear model with 6 Jacobi iterations.

In summary, the $k$ - $\varepsilon$ model is seen to perform well in a complex three-dimensional flow with only a small increase in CPU time required over the existing SpalartAllmaras model.

\section{Concluding Remarks}

A systematic study has been conducted to assess the accuracy of two newly implemented turbulence models; modified versions of the standard linear two-equation $k-\varepsilon$ model and the non-linear algebraic Reynolds stress model of Girimaji. Initial results of tests on the flat plate, airfoil, and wing cases indicate

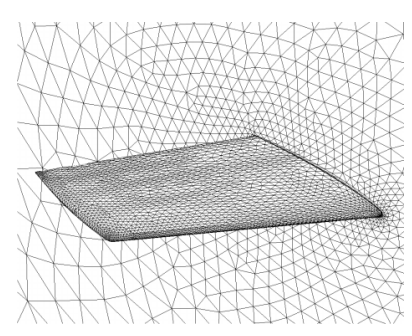

a)

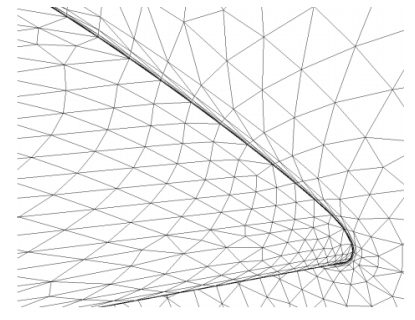

b)
Fig. 7 Surface mesh for a) entire wing and b) root region of symmetry plane.

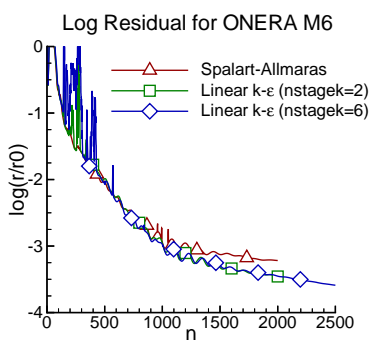

a)

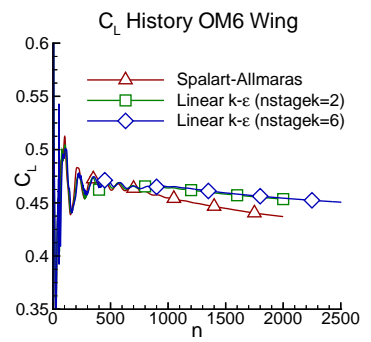

c)

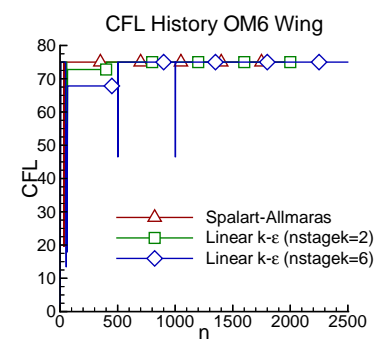

b)

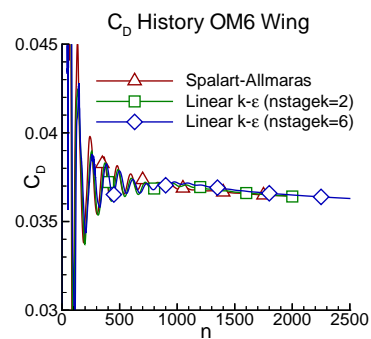

d)
Fig. 8 History of a) Log residual, b) CFL number, c) $C_{L}$ and d) $C_{D}$ for ONERA M6 wing.

OM6 USM3Dns Solution - 2000 cycles $\mathrm{M}=0.8447, \mathrm{AOA}=5.06, \mathrm{Re}=11.7 \mathrm{M}$

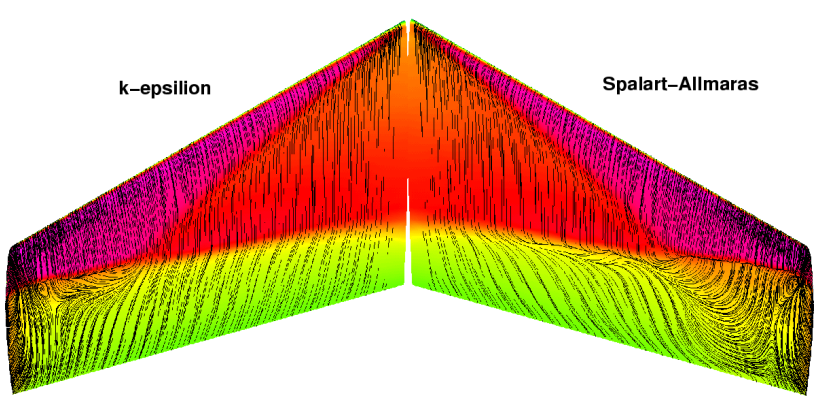

Fig. 9 Comparison of $k-\varepsilon$ (ivisc=7) and SpalartAllmaras model computed surface "oil-flow" patterns and flooded pressure contours for the ONERA M6 wing. 


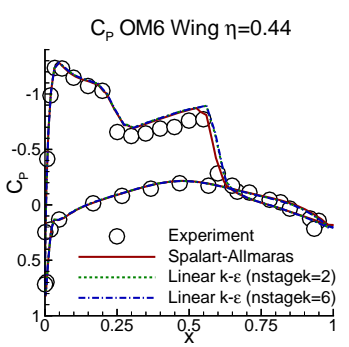

a) $\eta=0.44$

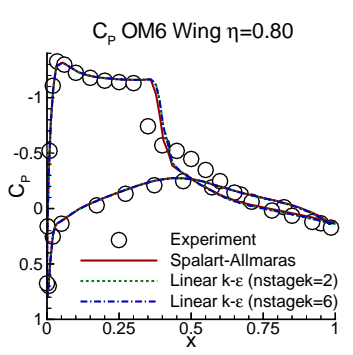

c) $\eta=0.80$

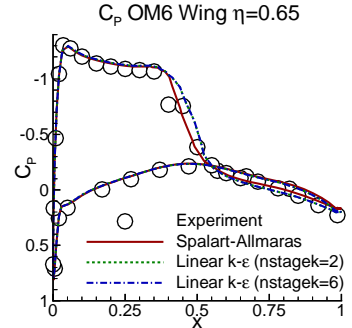

b) $\eta=0.65$

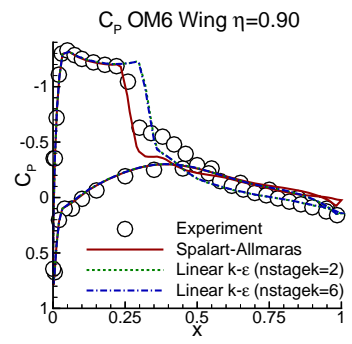

d) $\eta=0.90$
Fig. 10 Comparison of coefficient of pressure from each model $(n=2,000)$ with the experimental data of Schmitt ${ }^{28}$.

that the new two-equation models yield comparable accuracy and efficiency to that of the Spalart-Allmaras one-equation model. Work is currently underway to further examine factors such as grid sensitivities and solution details.

\section{Acknowledgements}

This research was supported by the Configuration Aerodynamics Branch of NASA Langley Research Center. Supplemental computational resources were also generously provided by CAB of LaRC.

\section{References}

${ }^{1}$ Mavriplis, D., "Unstructured-Grid Techniques," Ann. Rev. Fluid Mech., Vol. 29, 1997, pp. 473-514.

${ }^{2}$ Mavriplis, D., "Turbulent Flow Calculation Using Unstructured and Adaptive Meshes," Int. J. Numer. Meth. Fluids, Vol. 13, 1991, pp. 1131-1141.

${ }^{3}$ Baldwin, B. and Lomax, H., "Thin Layer Approximation and Algebraic Model for Separated Turbulent Flows," AIAA Paper 78-257.

${ }^{4}$ Mavriplis, D. and Martinelli, L., "Multigrid Solution of Compressible Turbulent Flow on Unstructured Mesh Using a Two-Equation Model," ICASE Technical Report 91-11.

${ }^{5}$ Anderson, W., "A Grid Generation and Flow Solution Method for the Euler Equations on Unstructured Grids," Journal of Computational Physics, Vol. 110, 1994, pp. 23-38.

${ }^{6}$ Anderson, W. and Bonhaus, D., "An implicit upwind algorithm for computing turbulent flows on unstructured grids," Computers and Fluids, Vol. 23, 1994, pp. 1-21.

${ }^{7}$ Baldwin, B. and Barth, T., "A One-Equation Turbulence Transport Model for High Reynolds Number Wall Bounded Flows," NASA Technical Memorandum 102847, 1978.

${ }^{8}$ Spalart, P. and Allmaras, S., "A One-Equation Turbulence Model for Aerodynamic Flows," AIAA Paper 92-0439, 1992.

${ }^{9}$ Frink, N., Parikh, P., and Pirzadeh, S., "A Fast Upwind Solver for the Euler Equations on Three-Dimensional Unstructured Meshes," AIAA Paper 91-0102, 1991.
${ }^{10}$ Frink, N., "Upwind Scheme for Solving the Euler Equations on Unstructured Tetrahedral Meshes," AIAA Journal, Vol. 30, 1992, pp. 70-77.

${ }^{11}$ Frink, N., "Recent Progress Toward a Three-Dimensional Unstructured Navier-Stokes Flow Solver," AIAA Paper 94-9961, 1994.

${ }^{12}$ Frink, N., "Assessment of an Unstructured-Grid Method for Predicting 3-D Turbulent Viscous Flows," AIAA Paper 960292, 1996.

${ }^{13}$ Kwon, O. and Hah, C., "Solution of the 3-D Navier Stokes Equations with a Two-Equation Turbulence Model on Unstructured Meshes Applied to Turbomachinery," AIAA Paper 941833, 1994.

${ }^{14}$ Wang, Q., Massey, S. J., and Abdol-Hamid, K. S., "Implementation of Advanced Two Equation Turbulence Models in the USM3D Unstructured Flow Solver," NASA Contractor Report xxxx, 1998, CR number not assigned before print deadline.

${ }^{15}$ Sarkar, S., Erlebacher, G., Hussaini, M., and Kreiss, H., "The Analysis and Modeling of Dilatational Terms in Compressible Turbulence," J. Fluid Mech., Vol. 227, 1991, pp. 473-495.

${ }^{16}$ Wilcox, D., "Progress in Hypersonic Turbulence Modeling," AIAA Paper 92-0439, 1991.

${ }^{17}$ Jones, W. and Launder, B., "The Prediction of Laminarization with a Two-Equation Model of Turbulence," Int. J. Heat Mass Transf., Vol. 15, 1972, pp. 301-314.

${ }^{18}$ Nagano, Y. and Hashida, M., "Improved Form of the $k-\varepsilon$ Model for Wall Turbulent Shear Flow," J. Fluid Mech., Vol. 109, 1987, pp. 156-160.

${ }^{19}$ Speziale, C., Abi, R., and Anderson, E., "A Critical Evaluation of Two-Equation Models for Near-Wall Turbulence," AIAA Paper 90-1481, 1990.

${ }^{20}$ Carlson, J. R., Personal communication, June 1998. Configuration Aerodynamics Branch, LaRC, Hampton, VA.

${ }^{21}$ Girimaji, S., "Fully-Explicit and Self-Consistant Algebraic Reynolds Stress Model," NASA CR 198243, 1995.

${ }^{22}$ Roe, P., "Characteristic Based Schemes for the Euler Equations," Ann. Rev. Fluid Mech., Vol. 18, 1986, pp. 337-365.

${ }^{23}$ Van Leer, B., "Flux-Vector Splitting for the Euler Equations," Lecture Notes in Physics, Vol. 170, 1982, pp. 507-512.

${ }^{24}$ Abdol-Hamid, K. S., Lashmanan, B., and Carlson, J. R., "Application of Navier-Stokes code PAB3D with $k-\varepsilon$ Turbulence Models to Attached and Separated Flows," NASA Technical Paper 3480, 1995.

${ }^{25}$ Pirzadeh, S., "Viscous Unstructured Three-Dimensional Grids by the Advancing-Layers Method," AIAA Paper 94-0417, 1994.

${ }^{26}$ Cook, P., McDonald, M., and Firman, M., "Airfoil RAE 2822-Pressure Distributions and Boundary Layer Wake Measurements," AGARD AR-138, May 1979.

${ }^{27}$ Rumsey, C. and Vatsa, V., "A Comparison of the Predictive Capabilities of Several Turbulence Models Using Upwind and Central-Difference Computer Codes," AIAA Paper 93-0192, 1993.

${ }^{28}$ Schmitt, V. and Charpin, F., "Pressure Distributions on the ONERA M6-Wing at Transonic Mach Number," AGARD AR-138, May 1979. 